\title{
Aportes para discutir el enfoque de las capacidades en la gestión de comunicación. Aplicación en Colombia
}

\section{Contributions to discuss the capabilities approach to communication management. Colombia's version}

\author{
Ana María Suárez-Monsalve \\ Carlos Alfonso López Lizarazo²
}

Resumen: La investigación que origina este artículo contribuye con la descripción de las capacidades en gestión de la comunicación para la práctica profesional en el contexto colombiano. Presenta los resultados de la consulta a profesionales y académicos de seis ciudades de Colombia, en dos rondas de análisis, empleando el método Delphi. De los enunciados expresados y validados por expertos se valoran capacidades profundamente humanas en la ética y el comportamiento bioético, capacidades de relacionamiento estratégico para la función social mediadora y de negociación, así como capacidades actitudinales y proactivas favorables a la gestión comunicacional en las organizaciones. De esta manera, se contribuye desde Colombia con el "Global Capabilities Framework" liderado por "Global Alliance for Public Relations and Communications Management". Palabras clave: comunicación; capacidades; ética; Colombia.

\footnotetext{
Abstract: This article contributes to the description of communication management capabilities for professional practice in the Colombian context. It

1 Universidad de Medellín. Medellín, Antioquia, Colombia. http://orcid.org/0000-0002-8611-6690 E-mail: asuarez@udemedellin.edu.co

2 Universidad de Medellín. Medellín, Antioquia, Colombia.

http://orcid.org/0000-0002-7724-5503 E-mail: calopez@udemedellin.edu.co
} 
presents the results of the research to professionals and academics from six cities in Colombia, in two rounds of analysis, using the Delphi method. Based on the statements expressed and validated by the experts, human capabilities in the area of ethics and bioethical behavior, strategic relationship capabilities for the social function of mediation and negotiation, as well as attitudinal and proactive capabilities in favor of communication management in organizations are evaluated. In this way, Colombia contributes to the Global Capabilities Framework led by the Global Alliance for Public Relations and Communications Management.

Key words: communication; capabilities; ethics; Colombia. 


\section{Introducción}

Los desafíos para la interacción, conciliación y transformación en la sociedad convocan a la revisión de los aportes que la comunicación, como ciencia social y como profesión, puede hacer en la formación individual y colectiva de sus practicantes. Uno de esos aportes urgentes es la identificación de las capacidades en gestión de la comunicación que deben fomentarse en la academia y en el mundo laboral. La condición de ciudadanos globales nos aboca a revisar los estándares de enseñanza para la apropiación tanto de los derechos como de los deberes con el mundo, partiendo desde la condición local para inscribirse en el ámbito global. Los escenarios de práctica profesional brindan oportunidad de incidencia en comportamiento y conductas favorables a la comunicación y al relacionamiento armónico que incidan en el entendimiento humano y la comprensión de las dinámicas socioculturales, ambientales, económicas y políticas que determinan la vida.

De acuerdo con SEN (1996a), se definen las capacidades como la habilidad de una persona para alcanzar estados valiosos de ser, pero las capacidades deben ser valoradas por los grupos con quienes se tiene interacción o filiación. Es decir, los deseos del individuo no son los únicos determinantes de las capacidades que éste debe desarrollar. Por eso, es necesario buscar el equilibrio entre los intereses individuales y las necesidades sociales. La academia y las asociaciones de profesionales actúan como mediadores para este equilibrio. La investigación que origina este artículo contribuye con esa discusión global.

\section{Contexto}

En las dos últimas décadas, en América Latina ha aumentado la investigación aplicada y teórica que cuestionan el poder de las organizaciones, así como la utilización de la información y los medios, tanto tradicionales como digitales, en esa dinámica. El debate incluye discusiones sobre las políticas económicas que impactan el sector organizacional empresarial, la nutrida incidencia de las organizaciones no gubernamentales 
en la región, así como la desconfianza en las decisiones corporativas y de gobierno ejecutivo en las naciones democráticas. Por eso, ha surgido una tendencia de estudio tanto en pregrado como en posgrados y doctorados de comunicación, donde se profundiza, con mirada crítica, temas como la responsabilidad de las organizaciones, tanto públicas como privadas, la transparencia de los actores sociales y políticos, la correspondencia en deberes de la ciudadanía corporativa y la gestión de la reputación, sobre la base de una economía que genere confianza.

Tal como lo reportaron PÉREZ; SUÁREZ (2017) en la conferencia "International Association for Mass Commmunication Research" (IAMCR) en Cartagena, Colombia, es posible afirmar que la gestión de la comunicación (incluyendo comunicación corporativa, comunicación organizacional y relaciones públicas) son un campo de estudio interdisciplinario que se ha fortalecido en los últimos años con la creación de doctorados en comunicación en Latinoamérica, así como mayor acceso a los ya creados en Europa y en Estados Unidos. Las disciplinas de estudio que han ayudado a reconfigurar el campo de la comunicación en América Latina son la sociología, la sicología, la filosofía, la economía y la administración. Esto ha llevado a configurar tres paradigmas desde los cuales se estudia hoy la gestión en comunicación: el sistémico (tradición norteamericana), el retórico (tradición norteamericana, europea y latinoamericana) y el crítico (tradición europea).

Para la investigación que da origen a este estudio, se tuvo en cuenta no sólo los enfoques teóricos sobre el desarrollo y las capacidades, sino el marco de la práctica profesional desde una condición más humana y social. Se parte de la necesidad de considerar un enfoque social acorde con las demandas del mundo actual (MOLLEDA et al, 2018), el papel de la academia y de las asociaciones de profesionales en la formación de capacidades de los individuos más allá de la aplicación a funciones y tareas en el campo profesional como lo propone la conceptualización de competencia. A continuación, se presenta un resumen de esta orientación teórica y la perspectiva profesional que se propone profundizar desde el enfoque de las capacidades. 
La federación de asociaciones profesionales "Global Alliance for $\mathrm{Pu}$ blic Relations and Communication Management", ("Global Alliance" en adelante), inició la revisión del enfoque de capacidades humanas en la comunicación entre los miembros (2018), motivó la aplicación de una consulta a profesionales y expertos en cada país donde existen programas académicos, así como un sector laboral en comunicación consolidado. A esta iniciativa se suman, progresivamente, otros países, académicos y asociaciones de comunicadores, con el fin de aportar a la discusión del enfoque en capacidades en los contextos propios, para nutrir también, mediante estudios comparativos, un cuerpo global de capacidades en gestión de la comunicación.

\section{El enfoque en las capacidades humanas}

El concepto de capacidad humana se refiere a la "habilidad" para llevar el tipo de vida que las personas consideran valiosa y de incrementar sus propias posibilidades de elección (SEN, 1996a). De otro lado, el capital humano se concentra en las habilidades, conocimiento y esfuerzos que aumentan las posibilidades de producción. Aunque ambos conceptos se agrupan en torno al ser humano, en el papel, en las habilidades que logran y adquieren, el autor señala cómo la capacidad humana es una perspectiva más amplia puesto que abarca las consecuencias directas e indirectas del capital humano.

En el escenario del desarrollo, las capacidades se concentran en el sentido de la humanidad y, como tal en el desarrollo social, mientras que el capital humano tiene un papel más restringido en la habilidad instrumental para la expansión de la productividad. El enfoque en las capacidades se dirige a la libertad que asume el individuo para dirigir mejor su vida y generar el cambio social.

Se puede entender que en el ejercicio de la libertad y de su papel de agente de cambio se incluyen, tanto como otros aspectos, los que conciernen al mejoramiento productivo del individuo, con una mayor educación, salud e, incluso, situación económica (SEN, 1996a). Pero la inversión de esta ecuación, con prioridad en el desarrollo del capital 
productivo, es lo que hace más pertinente analizar y priorizar la perspectiva del desarrollo centrado en las capacidades humanas, las capacidades para asumir y ejercer la libertad que motiven la transformación del desarrollo humano.

Para entender esta prioridad, hay que considerar el concepto de "funcionamiento". Los funcionamientos representan partes del estado de una persona, las cosas que logra hacer o ser al vivir; la calidad de vida debe evaluarse en términos de la capacidad para lograr acciones valiosas. De esta manera, para SEN (1996b) “algunos funcionamientos son muy elementales como estar nutrido adecuadamente, tener buena salud, etc. (...) Otros pueden ser más complejos, pero seguir siendo ampliamente apreciados como para alcanzar la autodignidad o integrarse socialmente", (1996b. Párr. 7). Por eso, señala el autor, es muy importante seleccionar los funcionamientos que son valiosos en los contextos en donde el ser humano debe elegir quién ser y qué hacer. Incluso, hay contextos en que el conjunto de problemas, además del económico, sugieren una lista extensa de funcionamientos importantes para describir y estimar las capacidades. Así, "el enfoque en las capacidades se basa en una visión de la vida en tanto combinación de quehaceres y seres, en los que la calidad de vida debe evaluarse en términos de la capacidad para lograr funcionamientos valiosos" (SEN, 1996b, Párr. 6). Es preciso hacer una selección de los temas y valores que subyacen en los contextos donde las personas serán evaluadas en términos de sus capacidades porque con esta selección se pueden identificar cuáles son los más importantes y los de menor relevancia.

Se debe considerar que las capacidades no sólo parten y se desarrollan por los deseos del individuo, sino que deben ser valoradas por los grupos con quienes se tiene interacción o filiación. Los deseos del individuo no son los únicos determinantes de las capacidades que se deben desarrollar. Por eso, hay que equilibrar los enfoques utilitaristas que consideran a los individuos solamente como un medio.

El enfoque de SEN (1996a), sobre el desarrollo humano puede ser utilizado en el análisis de las profesiones y, algunas asociaciones, lo 
han utilizado para la revisión de sus estándares de profesionalización. En este sentido, LESTER (2014), argumenta que las profesiones y la búsqueda de normas profesionales deben trascender las competencias, las habilidades y la acumulación de conocimiento práctico hacia fundamentaciones más significativas. Este autor apoya el enfoque de capacidad en la madurez profesional, ya que fomenta un compromiso más fluido y dinámico con cuestiones más amplias como la capacidad de reflexionar críticamente, de aplicar el juicio independiente en situaciones complejas y, más importantes que el conocimiento de códigos, la capacidad de utilizar la orientación ética en la toma de decisiones.

Por esto, las universidades, las asociaciones profesionales, los organismos públicos y privados deben cuestionarse en conjunto y constantemente sobre los funcionamientos (como se ha explicado atrás) o las fundamentaciones más significativas del contexto en el que los profesionales, como seres humanos, desarrollan sus capacidades. El diálogo constante sobre ese conjunto compartido de capacidades define el desarrollo humano y profesional a nivel mundial.

\section{Metodología y Resultados}

La federación de asociaciones profesionales "Global Alliance" promueve el estudio constante de los estándares educativos y la evaluación de los profesionales en gestión de la comunicación y las relaciones públicas en el mundo. Como federación de asociaciones en esta disciplina que tiene una red de 280.000 profesionales en el mundo, impulsó la revisión de un cuerpo global de conocimiento denominado "Global Body Of Knowledge”-GBOK- (MANELY; VALIN, 2017), que aportó una lista con descripciones sobre conocimientos, habilidades, atributos y comportamientos, según niveles de experiencia profesional -desde principiantes hasta experimentados-, producto del análisis de conjuntos o marcos de competencias en varios países y publicaciones científicas en el área de la comunicación. Este cuerpo global de conocimiento (GBOK), fue presentado en la conferencia anual de Global Alliance en 2018 y su publicación constituyó la base para las siguientes consultas 
con las demás asociaciones profesionales en el mundo. En el avance de esas discusiones, se ha cuestionado la adquisición y aplicación de esos conocimientos, habilidades, atributos y comportamientos (KSAB por sus siglas en inglés) para atender las necesidades del contexto particular en el momento específico del ejercicio profesional (GREGORY, 2008; MORENO, TENCH; OKAY, 2017). Derivado de las discusiones científicas y aplicadas por grupos de miembros asociados en Global Alliance, surgió una iniciativa adicional conocida como "Global Capability Framework for Public Relations and Communication Management", GCF, (FAWKES et al., 2018). Esta investigación, reunió a expertos académicos y profesionales para describir la profesión bajo el enfoque de las capacidades con la participación de investigadores de Argentina, Australia, Canadá, España, Estados Unidos, Reino Unido, Singapur, Suecia y Sudáfrica (GREGORY; FAWKES, 2019). La metodología fue cualitativa y cuantitativa, aplicada progresivamente, con la consulta a 1.400 expertos académicos y profesionales en varias rondas del método Delphi, grupos focales y encuestas, que concluyó con un listado de 11 capacidades en gestión de la comunicación y las relaciones públicas enunciadas y validadas por profesionales y académicos de estos nueve países.

Es así, como, en 2018, la Universidad de Medellín en Colombia, se vinculó como miembro de la "Global Alliance". Bajo la modalidad de investigación por cooperación interinstitucional y con el fin de incorporar el aporte de Colombia en el Cuerpo Global de Conocimiento, GCF, y con el antecedente de la aplicación en Argentina, incluida en el GCF se orientó con rigor metodológico para futuros estudios comparativos en América Latina. Adicionalmente, en 2018 también se aplicó el estudio en Ecuador, liderado por la Universidad Casa Grande y también con el acompañamiento de "Global Alliance" (BAQUERIZO-NEIRA; SADI, 2019). 


\section{La aplicación para el caso de Colombia en el GCF}

Entre 2018 y 2019, en Colombia se aplicaron dos fases de consulta Delphi a profesionales y expertos, un grupo focal y entrevistas individuales formales a directores de comunicación y académicos del país. La primera ronda del Delphi fue presentada a una base seleccionada de 50 profesionales y académicos. El diseño se guió del cuestionario original de Global Alliance con la metodología ajustada al español y aplicada en Argentina. La estructura explica una base conceptual de las capacidades y el cuestionario base se deriva de tres categorías para una primera definición y agrupación de las capacidades a consultar con los expertos. Esas primeras tres categorías son: Capacidad de Relacionamiento Estratégico (CRE), Capacidad de Comunicación Operativa (CCO), Capacidades Generales (CG).

Las preguntas dirigidas a los expertos se orientaban a la enunciación y agrupación entre 8 a 10 capacidades. En esta primera ronda 14 participantes respondieron la consulta: 5 de Bogotá, 5 de Medellín, 1 de Bucaramanga, 1 de Barranquilla, 1 de Montería y 1 de Sincelejo. Las acciones de las otras ciudades no llegaron en los plazos de entrega del primer informe. La participación del sector fue así: 3 educadores, 8 profesionales y 3 empleadores.

Una vez recogidos los cuestionarios resueltos de la primera ronda del Delphi, se procedió a identificar coincidencias por relación semántica de las capacidades sugeridas. En un primer listado, hecho con las respuestas de los expertos, se obtuvo un total de 142 enunciados.

La reducción de estos enunciados se hizo por el sentido semántico de las frases, agrupándolos en las categorías mencionadas antes.

En la categoría Capacidades de Relacionamiento Estratégico (CRE) se agruparon 66 enunciados; el de mayor coincidencia en esta categoría (con 14 referencias) se sintetizó en la capacidad de analizar, generar y sostener vínculos con aliados, contrapartes, stakeholders, grupos de interés, actores relevantes, sponsor, para articular, mediar, establecer acuerdos, de manera respetuosa, empática, constructiva y colectiva. 
En la categoría Capacidades de Comunicación Operativa (CCO) se recogen 43 enunciados, siendo la agrupación con mayor recurrencia (con 21) el que se refiere a la capacidad de dominio comunicacional desde las narrativas hasta la identificación del contenido, planeación estratégica de mensajes, medios, plataformas, audiencias y públicos, así como las capacidades propias de la comunicación como escucha, escritura, síntesis, dominio del español y de una segunda lengua, el inglés. Todo esto en relación con el contexto, situación y contenido comunicacional para el diálogo y la deliberación.

En la categoría Capacidades Generales (CG) se identificaron 33 enunciados, de los cuales, el que más recurrencia tuvo fue el referido a la capacidad de índole humano, personal o individual que recoge valores desarrollados como la empatía, la prudencia, la calidez, la disponibilidad, la cooperación, el equilibrio, el servicio y las buenas relaciones desde la comprensión del ser humano. Se recogen en la capacidad de entendimiento de la vida y del comportamiento bioético, es decir impactando positivamente los grupos humanos y la naturaleza.

Después de un proceso de lectura y relectura, se redujeron los enunciados, se agruparon nuevamente para así categorizar por sentido. Posteriormente, en la discusión del grupo focal se propusieron tres enunciados adicionales que se pusieron a consideración en la segunda ronda del Delphi y así, se concretaron 14 capacidades, tal como se aprecia a continuación: 
Tabla 1. Enunciados sobre capacidades en gestión de la comunicación depurados por validación del Grupo Focal ronda 1.

\begin{tabular}{|c|c|c|}
\hline $\begin{array}{l}\text { Numeración } \\
\text { inicial de la } \\
\text { capacidad }\end{array}$ & Enunciado que integra la Capacidad & $\begin{array}{l}\text { Frecuencia de } \\
\text { repetición del } \\
\text { enunciado }\end{array}$ \\
\hline 3 & $\begin{array}{l}\text { Capacidad narrativa, planeación estratégica de } \\
\text { mensajes, medios, plataformas, audiencias y } \\
\text { públicos, así como las capacidades propias de la } \\
\text { comunicación como escucha, escritura, síntesis, } \\
\text { dominio del español y de una segunda lengua, el } \\
\text { inglés. Todo en relación con el contexto, situación } \\
\text { y contenido comunicacional para el diálogo y la } \\
\text { deliberación }\end{array}$ & 21 \\
\hline 8 & $\begin{array}{l}\text { Capacidad de analizar, generar y sostener vínculos } \\
\text { con aliados, contrapartes, stakeholders, grupos de } \\
\text { interés, actores relevantes, sponsor, para articular, } \\
\text { mediar, establecer acuerdos, de manera respetuo- } \\
\text { sa, empática, constructiva y colectiva" }\end{array}$ & 19 \\
\hline 1 & $\begin{array}{l}\text { Capacidad de liderazgo con decisión para ejercer } \\
\text { la gestión de procesos comunicativos con planea- } \\
\text { ción, coordinación y evaluación de estrategias, } \\
\text { programas y proyectos con que generen retorno a } \\
\text { la inversión. }\end{array}$ & 14 \\
\hline 9 & $\begin{array}{l}\text { Capacidad para desarrollar la empatía, la crea- } \\
\text { tividad, la solidaridad y la comprensión del ser } \\
\text { humano, para mejor entendimiento de la vida y } \\
\text { tener un comportamiento bioético, impactando } \\
\text { positivamente los grupos humanos y la naturaleza. }\end{array}$ & 14 \\
\hline 5 & $\begin{array}{l}\text { Capacidad de observar, reflexionar, analizar y } \\
\text { comprender el entorno e incidir sobre el mismo } \\
\text { buscando el bienestar de los grupos de interés. }\end{array}$ & 11 \\
\hline 7 & $\begin{array}{l}\text { Capacidad de mediación, negociación, de concer- } \\
\text { tación, de resolución de conflictos y de incidencia } \\
\text { tanto para posicionar la comunicación como para } \\
\text { propósitos colectivos }\end{array}$ & 10 \\
\hline 11 & $\begin{array}{l}\text { Capacidad para la previsión de riesgos, la adapta- } \\
\text { bilidad eficiente al cambio, la caracterización y } \\
\text { la actitud positiva ante las crisis y, en general, las } \\
\text { distintas situaciones que se presenten con criterios } \\
\text { profesionales y morales }\end{array}$ & 9 \\
\hline
\end{tabular}




\begin{tabular}{|c|c|c|}
\hline $\begin{array}{l}\text { Numeración } \\
\text { inicial de la } \\
\text { capacidad }\end{array}$ & Enunciado que integra la Capacidad & $\begin{array}{l}\text { Frecuencia de } \\
\text { repetición del } \\
\text { enunciado }\end{array}$ \\
\hline 6 & $\begin{array}{l}\text { Capacidad de análisis de oportunidades, riesgos, } \\
\text { amenazas, de comportamientos de la opinión } \\
\text { pública y de contenidos relevantes, para pla- } \\
\text { nificar estrategias como para generar espacios } \\
\text { donde se construya ese análisis, el discernimiento } \\
\text { entre resultados e impacto del campo específico } \\
\text { comunicacional. }\end{array}$ & 9 \\
\hline 4 & $\begin{array}{l}\text { Capacidad para el pensamiento estratégico, para } \\
\text { establecer criterios de valoración y acción puntual } \\
\text { al desarrollo de estrategias de manera holística }\end{array}$ & 8 \\
\hline 2 & $\begin{array}{l}\text { Capacidad para actualizarse y manejar herramien- } \\
\text { tas técnicas y tecnológico de plataformas, redes, } \\
\text { medios y canales. }\end{array}$ & 7 \\
\hline 10 & $\begin{array}{l}\text { Capacidad para el trabajo en equipo, la interdis- } \\
\text { ciplinariedad y la articulación con las diferentes } \\
\text { unidades de las empresas, generando confianza. }\end{array}$ & 7 \\
\hline 12 & $\begin{array}{l}\text { Capacidad de aprendizaje y des-aprendizaje, } \\
\text { proposición, creatividad, dinamismo e innovación } \\
\text { para el emprendimiento y para la resiliencia }\end{array}$ & 6 \\
\hline 13 & $\begin{array}{l}\text { Capacidad para la gestión del conocimiento, } \\
\text { medición y evaluación apoyados en las técnicas de } \\
\text { investigación. }\end{array}$ & 5 \\
\hline 14 & $\begin{array}{l}\text { Capacidad ética de la profesión y de la organiza- } \\
\text { ción a la cual le sirve con compromiso, valores y } \\
\text { persistencia }\end{array}$ & 2 \\
\hline TOTAL & 14 Capacidades & $\begin{array}{l}142 \\
\text { enunciados }\end{array}$ \\
\hline
\end{tabular}

Fuente: Elaboración propia.

Con este listado de 14 capacidades, se solicitó a los participantes del Delphi en la ronda 1 que jerarquizaran las capacidades agrupadas y las calificaran en una escala Likert de menor a mayor, siendo 1 no importante y 7 de extrema importancia. En esta revisión y valoración se obtuvo respuesta por 20 profesionales y expertos de las principales ciudades del país, así: 


\begin{tabular}{|c|c|c|c|c|c|c|}
\hline م芯芯芯 & $=$ & \pm & $\stackrel{m}{-}$ & $a$ & $\stackrel{m}{=}$ & $a$ \\
\hline 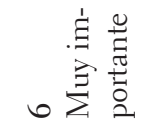 & $\Lambda$ & + & n & $\Lambda$ & $\Lambda$ & $\varrho$ \\
\hline 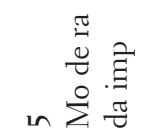 & 一 & $\sim$ & $\sim$ & $m$ & & 一 \\
\hline $\begin{array}{r}\underset{\Xi}{\Xi} \\
+Z \\
\end{array}$ & & & & 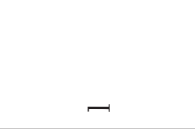 & & \\
\hline 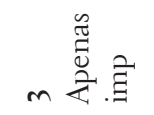 & - & & & & & \\
\hline 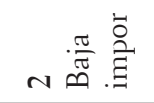 & & & & & & \\
\hline 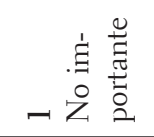 & & & & & & \\
\hline 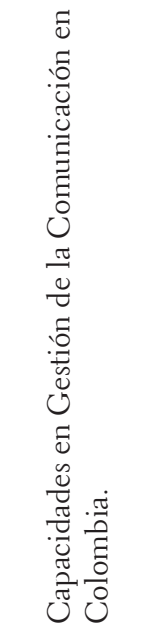 & 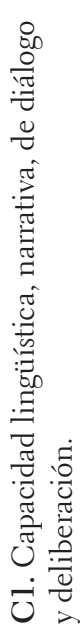 & 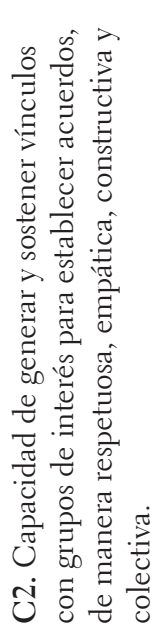 & 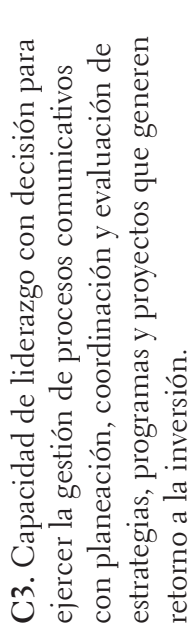 & 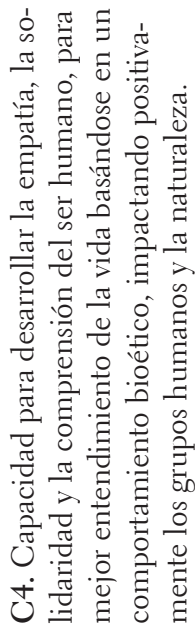 & 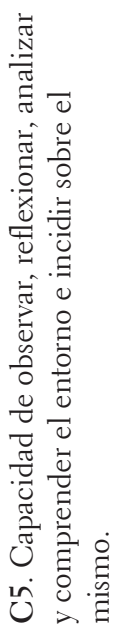 & 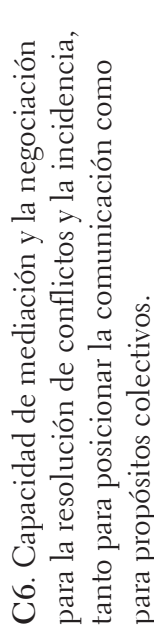 \\
\hline
\end{tabular}


ANA MARÍA SUÁREZ-MONSALVE | CARLOS ALFONSO LÓPEZ LIZARAZO 571

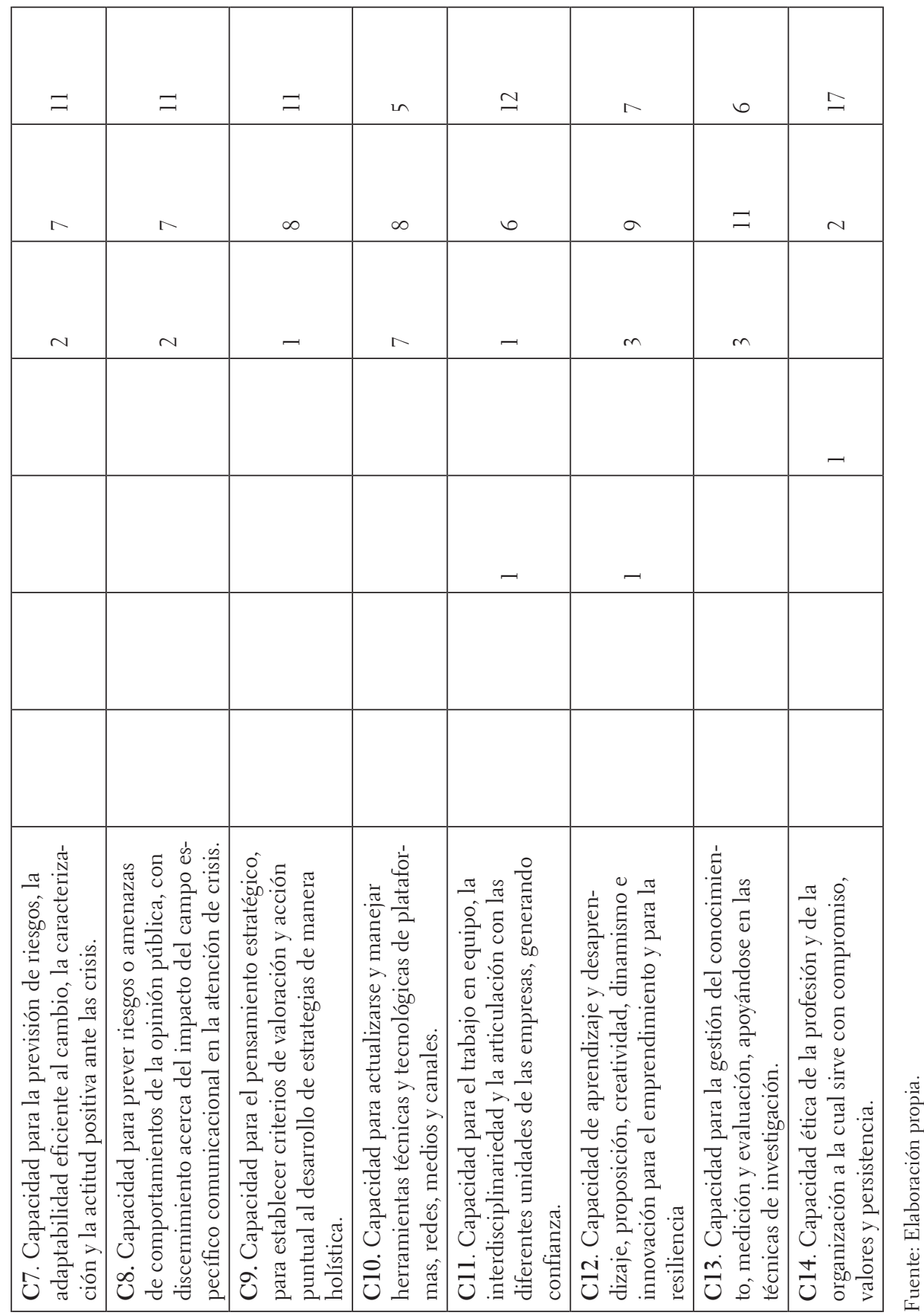


Así, se identifica una priorización por importancia para el contexto colombiano, según el valor dado en la escala de Likert, expresado en este orden:

1. "La capacidad ética de la profesión y de la organización a la cual sirve con compromiso, valores y persistencia", tiene la mayor valoración: fue calificada "De extrema importancia" 17 veces señalada por los profesionales, 2 veces votada como "Muy importante" y l vez asignada como "Neutral".

2. "La capacidad de generar y sostener vínculos con grupos de interés para establecer acuerdos, de manera respetuosa, empática, constructiva y colectiva" tiene la segunda mayor valoración "De extrema importancia" con 14 veces señalada por los profesionales, 4 veces votada como "Muy importante" y 2 veces señalada como de "Moderada importancia”.

3. "La capacidad de observar, reflexionar, analizar y comprender el entorno e incidir sobre el mismo" tiene la tercera mayor valoración "De extrema importancia” con 13 veces votada. Esta valoración también la obtuvo la capacidad siguiente, pero se identifica que, en ésta, se considera también "Muy importante" para el resto de los profesionales, con 7 votos.

4. "La capacidad de liderazgo con decisión para ejercer la gestión de procesos comunicativos con planeación, coordinación y evaluación de estrategias, programas y proyectos que generen retorno a la inversión” tiene también la tercera mayor valoración "De extrema importancia” con 13 veces votada. Sin embargo, los votos restantes se distribuyen en "Muy importante" 5 veces y de "Moderada importancia" 2 veces.

5. "La capacidad para el trabajo en equipo, la interdisciplinariedad y la articulación con las diferentes unidades de las empresas, generando confianza" tiene la cuarta mayor valoración "De extrema importancia" con 12 votos, "Muy importante" con 6 votos, "Moderada importancia" obtuvo 1 voto y también 1 voto como "Apenas importante". 
6. "La capacidad para el pensamiento estratégico, para establecer criterios de valoración y acción puntual al desarrollo de estrategias de manera holística" tiene 11 votos en la consideración de "Extrema importancia", le siguen 8 votos en "Muy importante" y 1 voto en "Moderada importancia".

7. "La capacidad para la previsión de riesgos, la adaptabilidad eficiente al cambio, la caracterización y la actitud positiva ante las crisis" tiene una valoración "De extrema importancia" con 11 votos, "Muy importante" con 7 votos y de "Moderada importancia" con 2 votos.

8. Igual que en la anterior, "La capacidad para prever riesgos o amenazas de comportamientos de la opinión pública, con discernimiento acerca del impacto del campo específico comunicacional en la atención de crisis" tiene una valoración "De extrema importancia” con 11 votos, "Muy importante" con 7 votos y de "Moderada importancia" con 2 votos.

9. "La capacidad lingüística, narrativa, de diálogo y deliberación" es considerada "De extrema importancia" por 11 votos, "Muy importante" con 7 votos, "De moderada importancia" con 1 voto y "Apenas importante" con 1 voto.

10. "La capacidad de mediación y la negociación para la resolución de conflictos y la incidencia, tanto para posicionar la comunicación, como para propósitos colectivos" tiene 9 votos como "De extrema importancia", 10 votos como "Muy importante" y 1 voto con "Moderada importancia”.

11. "La capacidad para desarrollar la empatía, la solidaridad y la comprensión del ser humano, para mejor entendimiento de la vida basándose en un comportamiento bioético, impactando positivamente los grupos humanos y la naturaleza" obtuvo 9 votos en la consideración "De extrema importancia", 7 votos en "Muy importante", 3 votos en de "Moderada importancia" y 1 voto como "Neutral".

12. "La capacidad de aprendizaje y desaprendizaje, proposición, creatividad, dinamismo e innovación para el emprendimiento y para la resiliencia" fue evaluada con 7 votos como "De extrema importancia”, 
con 9 votos como "Muy importante", con 3 votos con "Moderada importancia" y con 1 voto "Apenas importante".

13. "La capacidad para la gestión del conocimiento, medición y evaluación, apoyándose en las técnicas de investigación” fue valorada como "De extrema importancia" con 6 votos; "Muy importante" con 11 votos, de "Moderada importancia" con 3 votos.

14. “La capacidad para actualizarse y manejar herramientas técnicas y tecnológicas de plataformas, redes, medios y canales" obtuvo una valoración de 5 votos como "De extrema importancia", con 8 votos "Muy importante" y con 3 votos de "Moderada importancia".

De esta manera, la valoración más consistente está en la ética y el compromiso profesional, capacidad profundamente humana para guiar la actuación profesional. Le sigue en importancia la capacidad estratégica relacional para generar y sostener vínculos, el análisis e incidencia en el entorno, así como la capacidad estratégica comunicacional con el liderazgo de los procesos de planeación, coordinación y evaluación de la gestión en comunicación, en relación directa con el retorno a la inversión.

También se valora una capacidad que deriva de estas dos últimas, tanto lo relacional como la coordinación de la gestión, es la que resalta el trabajo en equipo interdisciplinario que genera confianza con las diferentes unidades organizativas. Como tal, se puede entender que esta es una derivación de las tres capacidades mencionadas en el párrafo anterior, las más valoradas, tal como se expresan en la condición humana, la capacidad analítica estratégica del entorno, en la capacidad estratégica relacional y de gestión comunicacional. Así mismo, la capacidad para el pensamiento estratégico se relaciona con el análisis del entorno ya mencionado para desarrollar acciones que se deriven de ese análisis.

Se observa con atención que la capacidad para hacer frente al riesgo tiene dos connotaciones, valoradas de manera similar: una interna, del individuo, de índole actitudinal. También, desde el punto de vista profesional, con el conocimiento sobre el comportamiento de la opinión pública y las acciones-procedimientos de atención de las posibles crisis. 
Adicionalmente, se destaca la capacidad orientada al servicio, a la función social de la comunicación y el relacionamiento que expresa la mediación y la negociación, evidenciada en la valoración promedio entre el valor máximo y el anterior, así como en la poca dispersión en la evaluación de esta capacidad.

Dos capacidades más hacen referencia a la condición humana relacionadas con la ética (más valorada) que son la capacidad de comprensión del ser humano y el comportamiento bioético, y la capacidad proactiva frente al aprendizaje, innovación y resiliencia.

Otro grupo de capacidades que se puede identificar, con valoración similar como "Muy importante", pero que presenta más dispersión en los valores otorgados por los profesionales, son las referidas a capacidades profesionales de dominio del lenguaje y habilidades comunicativas, manejo de herramientas técnicas-tecnológicas, plataformas- medios, y la gestión del conocimiento, evaluación e investigación.

\section{En sintesis}

Con este análisis, de acuerdo con la priorización dada por los valores y la consistencia de éstos, las capacidades en gestión de la comunicación en Colombia, según la muestra descrita antes en seis ciudades del país y la metodología aplicada, muestran que las capacidades se ordenan por valoración de extrema importancia a menor importancia y por número de enunciados que las citan, dando en el siguiente orden para el caso colombiano: 
Tabla No. 3. Cuerpo de capacidades en gestión de la comunicación identificadas en Colombia.

\begin{tabular}{|c|c|}
\hline 1. & $\begin{array}{l}\text { La capacidad ética de la profesión y de la organización a la cual sirve } \\
\text { con compromiso, valores y persistencia. }\end{array}$ \\
\hline 2. & $\begin{array}{l}\text { La capacidad de generar y sostener vínculos con grupos de interés } \\
\text { para establecer acuerdos, de manera respetuosa, empática, construc- } \\
\text { tiva y colectiva }\end{array}$ \\
\hline 3. & $\begin{array}{l}\text { La capacidad de observar, reflexionar, analizar y comprender el } \\
\text { entorno e incidir sobre el mismo. }\end{array}$ \\
\hline 4. & $\begin{array}{l}\text { La capacidad de liderazgo con decisión para ejercer la gestión de pro- } \\
\text { cesos comunicativos con planeación, coordinación y evaluación de } \\
\text { estrategias, programas y proyectos que generen retorno a la inversión. }\end{array}$ \\
\hline 5. & $\begin{array}{l}\text { La capacidad para el trabajo en equipo, la interdisciplinariedad y la } \\
\text { articulación con las diferentes unidades de las empresas, generando } \\
\text { confianza. }\end{array}$ \\
\hline 6. & $\begin{array}{l}\text { La capacidad para el pensamiento estratégico, para establecer cri- } \\
\text { terios de valoración y acción puntual al desarrollo de estrategias de } \\
\text { manera holística. }\end{array}$ \\
\hline 7. & $\begin{array}{l}\text { La capacidad para la previsión de riesgos, la adaptabilidad eficiente al } \\
\text { cambio, la caracterización y la actitud positiva ante las crisis. }\end{array}$ \\
\hline 8. & $\begin{array}{l}\text { La capacidad para prever riesgos o amenazas de comportamientos de } \\
\text { la opinión pública, con discernimiento acerca del impacto del campo } \\
\text { específico comunicacional en la atención de crisis. }\end{array}$ \\
\hline 9. & La capacidad lingüística, narrativa, de diálogo y deliberación. \\
\hline 10. & $\begin{array}{l}\text { La capacidad de mediación y la negociación para la resolución de } \\
\text { conflictos y la incidencia, tanto para posicionar la comunicación, } \\
\text { como para propósitos colectivos. }\end{array}$ \\
\hline 11. & $\begin{array}{l}\text { La capacidad para desarrollar la empatía, la solidaridad y la compren- } \\
\text { sión del ser humano, para mejor entendimiento de la vida basándose } \\
\text { en un comportamiento bioético, impactando positivamente los } \\
\text { grupos humanos y la naturaleza. }\end{array}$ \\
\hline 12. & $\begin{array}{l}\text { La capacidad de aprendizaje y desaprendizaje, proposición, creati- } \\
\text { vidad, dinamismo e innovación para el emprendimiento y para la } \\
\text { resiliencia. }\end{array}$ \\
\hline 13. & $\begin{array}{l}\text { La capacidad para la gestión del conocimiento, medición y evalua- } \\
\text { ción, apoyándose en las técnicas de investigación. }\end{array}$ \\
\hline 14. & $\begin{array}{l}\text { La capacidad para actualizarse y manejar herramientas técnicas y } \\
\text { tecnológicas de plataformas, redes, medios y canales. }\end{array}$ \\
\hline
\end{tabular}

Fuente: Elaboración propia. 


\section{Discusión de resultados}

El "Global Body Of Knowledge" entrega un marco de capacidades fundamentado en la expresión de las necesidades en los contextos europeos y norteamericano, como lo señalan GUTIÉRREZ; SADI (2020), con un marcado sesgo anglosajón, según los autores, pues esas regiones tuvieron mayor representación por países, mientras que por Asia, África y América Latina solo se contó con 1 país participante en este primer cuerpo global de capacidades (GCF) aplicado a la comunicación. Ahora bien, por América Latina la representación que logró inicialmente Argentina, robustece el GCF, con los matices propios de una región.

No obstante, se han sumado otros países que aplican la metodología expuesta por GREGORY; FAWKES (2019) pues aplican las técnicas de investigación y análisis en los contextos propios, como Ecuador (BARQUERIZO; SADI, 2019) para sumar por Latinoamérica en los estudios comparativos, hasta configurar más ampliamente el marco global de las capacidades en gestión de la comunicación (GREGORY; FAWKES, 2019; GUTIÉRREZ-GARCÍA; SADI, 2020). Ahí, es dónde Colombia también contribuye con una perspectiva propia de las capacidades como expresión de la libertad para la toma de decisiones en contexto, según la formación del ser y la condición humana, en relación con la gestión de la comunicación hacia la formación de nuevos profesionales. Sin embargo, en Colombia, se ha estudiado con mayor profundidad las competencias como habilidades para saber hacer. Es claro que, al preguntarse por las capacidades, de lo que se habla hoy es de saber ser, dado que es a partir de los funcionamientos vitales que se orientan vocaciones, procesos formativos y, finalmente, el ejercicio profesional en contextos locales de desigualdad articuladas a problemáticas humanas globales.

\section{Conclusiones y Recomendaciones}

Las capacidades referidas a la condición humana para los profesionales en gestión de la comunicación se concentran en la ética, el análisis del 
contexto, el trabajo en equipo, la mediación y negociación, la adaptabilidad, la actitud positiva, la solidaridad y la comprensión del ser humano basándose en el comportamiento bioético. De igual manera, las capacidades específicas de la profesión se concentran en el generar y sostener vínculos a largo plazo, la articulación de intereses y la previsión de riesgos comunicacionales.

Este es un aporte a la discusión de las capacidades globales en gestión de la comunicación desde Colombia, para sumar al cuerpo global de capacidades (GCF) promovido por "Global Alliance". Sin embargo, es necesario profundizar más en las capacidades para la gestión de la comunicación que incidan en el contexto social, político y económico colombiano, toda vez que estos generan influencia en la práctica profesional. Se requiere un ser humano con libertad y autonomía en el ejercicio laboral con potencialidades para ejercer su labor comunicacional con principios de comportamiento ético.

Las limitaciones previsiblemente consideradas por una metodología cualitativa hacen necesaria la aplicación de muestras mixtas en un futuro, que contribuyan con nuevos elementos del contexto y así, profundizar en la constante o variación de estas capacidades enunciadas. En futuros reportes, se profundizará en las comparaciones por países de América Latina con el fin de trazar una línea de discusión regional que ponga en discusión las variables del contexto para motivar la formación en capacidades para la gestión de comunicación con mayor pertinencia, según las dinámicas sociales, políticas y económicas que expresan las culturas latinoamericanas.

\section{Referencias}

BARQUERIZO, G.; SADI, G. Global Capabilities Framework. Aplicación en Ecuador de un estudio global sobre las capacidades del profesional de las relaciones públicas. Trípodos, Barcelona, n. 45, p. 13-29, 2019.

FAWKES, J.; GREGORY, A.; FALKHEIMER, J.; GUTIÉRREZ-GARCÍA, E.; HALFF, G.; RENSBURG, R.; WOLF, K. A. Global Capability Framework for the Public Relations and Communication Management profession. Global Alliance for Public Relations and Communication Management Research Report. Huddersfield, UK: University of 
Huddersfield, 2018. Disponible en: https://www.globalalliancepr.org. Acceso en: 19 dic. 2019.

GLOBAL ALLIANCE FOR PUBLIC RELATIONS AND COMMUNICATION MANAGEMENT. Disponible en: https://www.globalalliancepr.org. Acceso en: 21 jul. 2019.

GREGORY, A. Y FAWKES, J. A global capability framework: Reframing public relations for a changing world. Public Relations Review, v. 45, n. 3, sep. 2019. Disponible en: https://doi.org/10.1016/j.pubrev.2019.05.002. Acceso en: 30 jul. 2019.

GUTIÉRREZ-GARCÍA, E.; SADI, G. Capacidades profesionales para el mañana de la comunicación estratégica: contribuciones desde España y Argentina. Revista de Comunicación, v.19, n. 1, 2020. Disponible en: https://doi.org/10.26441/RC19.1-2020-A8. Acceso en: 5 may. 2020.

LESTER, S. Professional standards, competence and capability. Higher Education, Skills ๒ Work-Based Learning, v. 4, n. 1, p. 31-43, 2014. Disponible en: http://www. emeraldinsight.com/doi/abs/10.1108/HESWBL-04-2013-0005. Acceso en: dic 11. 2019. MOLlEDA, J. C.; SUÁREZ MONSALVE, A. M.; ATHAYDES, A.; SADI, G.; HERNÁNDEZ, E.; VALENCIA, R. Influences of postcolonialism over the understanding and evolution of public relations in Latin America. In: BRIDGEN, E; VERCIC, D. (Ed.). Experiencing Public Relations International Voices. p. 152-165. New York: Routledge, 2018.

MANLEY, D.; VALIN, J. Laying the foundation for a global body of knowledge in public relations and communications management. Public Relations Review, v. 43, n. 1, p. 56-70, 2017. Disponible en: https://doi. org/10.1016/J.PUBREV.2016.10.018. Acceso en: 10 may. 2019.

MORENO, A.; TENCH, R.; OKAY, A. Re-fuelling the talent tank. A qualitative study of key deficiencies, future needs, and life-long learning needs of communication management professionals in Europe. Communication ש Society, v. 30, n. 3, p. 109-127, 2017. SEN, A. Capital humano y capacidad humana. Cuadernos de Economía, v. 29, 1996 a. En: World Development . Traducción de Clara Ramírez. Disponible en: https:/revistas. unal.edu.co/index.php/ceconomia/article/view/1 1496/20791. Acceso en: 12 may. 2019. SEN, A. Capacidad y Bienestar, 1996b. Disponible en: http://www.eumed.net/cursecon/economistas/textos/Sen-capacidad_y_bienestar.htm Acceso en: 7 jun. 2018. 
Ana María Suárez-Monsalve - Doctora en Estudios Latinoamericanos Universidad de Chile, Magister en Educación en Educación Pontificia Universidad Javeriana, Comunicadora Social-Periodista Universidad de Antioquia. Profesora e investigadora de la Universidad de Medellín. Como investigadora principal dirigió la investigación, la construcción del marco teórico y elaboración de categorías conceptuales, el análisis de los datos, las conclusiones y la redacción del informe del proyecto para el artículo científico.

Carlos Alfonso López Lizarazo - Doctor en Estudios Latinoamericanos, Universidad de Chile. Magister en Educación en Educación con énfasis en Educación Superior, Pontificia Universidad Javeriana. Profesor e investigador de la Universidad de Medellín. Especialista en Dramaturgia, Universidad de Antioquia. Comunicadora Social, Universidad de Antioquia. Como coinvestigador aplicó técnicas de investigación y la sistematización de los datos, acompañó el proceso de diseño de análisis y la redacción del informe del proyecto para el artículo.

Data de submissão: 31/07/2020

Data de aceite: 03/12/2020 